\title{
CARACTERIZACIÓN DE LA INFESTACIÓN DE VIVIENDAS POR TRES ESPECIES DE MOSQUITOS (DIPTERA: CULICIDAE) DE IMPORTANCIA MÉDICA EN PAIISES DE BAJOS Y MEDIOS INGRESOS: RECOMENDACIONES PARA SU CONTROL DOMÉSTICO
}

\author{
Characterization of dwellings' infestation by three mosquito species \\ (Diptera: Culicidae) of medical importance: recommendations for its domestic \\ control in low- and middle-income countries
}

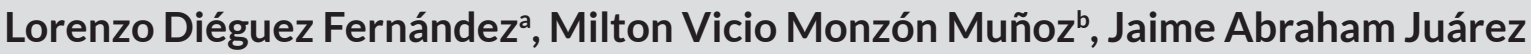 \\ Sandovalc, Dex Yorman Barrios Barrios ${ }^{\mathrm{d}}$, Mónica Elisa Barrientos Juárez ${ }^{\mathrm{e}}$, Yisel Hernández \\ Barrios $^{f}$, María Altagracia Rodríguez-Sosag, Yohan Enmanuel Vásquez-Bautistah \\ y Pedro María Alarcón-Elbali
}

Recibido: 6 de abril, $2021 \bullet$ Aprobado: 12 de junio, 2021

Cómo citar: Diéguez Fernández L, Monzón Muñoz MV, Juárez Sandoval JA, Barrios Barrios DY, Barrientos Juárez ME, Barrios YH, Rodríguez-Sosa MA, Vásquez-Bautista YE, Alarcón-Elbal PM. Caracterización de la infestación de viviendas por tres especies de mosquitos (Diptera: Culicidae) de importancia médica en países de bajos y medios ingresos: recomendaciones para su control doméstico. cysa [Internet]. 12 de octubre de 2021 [citado 15 de octubre de 2021];5(3):49-6. Disponible en: https://revistas.intec.edu.do/index. php/cisa/article/view/2307

\section{Resumen}

Entre las especies de mosquitos (Diptera: Culicidae) sinantrópicos que existen en la región de las Américas, tres desempeñan un papel protagónico en la transmisión de enfermedades de importancia médica en países de bajos y medios ingresos (LMIC): Aedes aegypti, Aedes albopictus y Culex quinquefasciatus. Con el objetivo de caracterizar la infestación domiciliar de estos mosquitos, se realizó un estudio entomológico en el municipio de Jutiapa (Guatemala) y Jarabacoa (República Dominicana), ambos incluidos

\footnotetext{
a Investigador Auxiliar del Laboratorio Provincial de Entomología Médica del Departamento de Control de Vectores, Centro Provincial de Higiene, Epidemiología y Microbiología de Camagüey, Cuba. ORCID: 0000-0002-5267-9575,

Correo-e: lorenzodieguez95@gmail.com

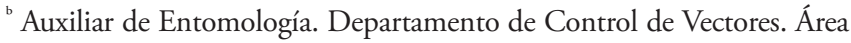
de Salud de Jutiapa. Ministerio de Salud Pública y Asistencia Social de Guatemala. Correo-e: vec22jutiapa@gmail.com

c Consultor Nacional de Eliminación de la Malaria en el Banco Interamericano de Desarrollo (BID) dentro de la iniciativa de eliminación de la malaria en Mesoamérica y la isla de La Española (IREM), Guatemala. Correo-e: jaimeju@iadb.org

d Profesor de Enseñanza Media en la Especialidad en Química y Biología. Sección Entomología Médica. Programa Enfermedades Transmitidas por Vectores. Ministerio de Salud Pública y Asistencia Social de Guatemala. Correo-e: entomedcentral@gmail.com
}

Among the synanthropic mosquito species (Diptera: Culicidae) that exist in the Americas region, three play a leading role in the transmission of diseases of medical importance in low- and middle-income countries: Aedes aegypti, Aedes albopictus and Culex quinquefasciatus. In order to characterize the household infestation of these mosquitoes, an entomological study was conducted in the municipalities of Jutiapa (Guatemala) and Jarabacoa (Dominican Republic),

\footnotetext{
e Programa Enfermedades Transmitidas por Vectores. Ministerio de Salud Pública y Asistencia Social de Guatemala.

Correo-e: monica.barrientos@mspas.gob.gt

f Investigadora y Profesora del Departamento Control de Vectores, Instituto de Medicina Tropical Pedro Kourí (IPK). La Habana, Cuba. Correo-e: yhbarrios@ipk.sld.cu

g Ingeniera Agrónoma y Asistente de Investigación en el Laboratorio de Entomología, Universidad Agroforestal Fernando Arturo de Meriño (UAFAM). Jarabacoa, República Dominicana.

Correo-e: a.investigacion1@uafam.edu.do

h Licenciado en Educación y Asistente de Investigación en el Laboratorio de Entomología, Universidad Agroforestal Fernando Arturo de Meriño (UAFAM). Jarabacoa, República Dominicana.

Correo-e: a.investigacion2@uafam.edu.do

Investigador en el Laboratorio de Entomología, Universidad Agroforestal Fernando Arturo de Meriño (UAFAM). Jarabacoa, República Dominicana. Correo-e: pedro.alarcon@uv.es
} 


\section{Lorenzo Diéguez Fernández, Milton Vicio Monzón Muñoz, Jaime Abraham Juárez Sandoval, Dex Yorman Barrios \\ Barrios, Mónica Elisa Barrientos Juárez, Yisel Hernández Barrios, María Altagracia Rodríguez-Sosa, Yohan Enmanuel Vásquez-Bautista y Pedro María Alarcón-Elbal}

dentro de la clasificación de LMIC. En cada municipio se muestrearon 100 viviendas seleccionadas aleatoriamente; en cada criadero se capturó la mayor cantidad posible de larvas y/o pupas con la ayuda de pipetas Pasteur, bandejas plásticas y botes herméticos. El porcentaje de depósitos con agua infestados por alguna de las especies de culícidos estudiada fue similar en ambos municipios (Jutiapa $=27.9$ $\%$, Jarabacoa $=20.0 \%$; ; entre estos predominaron los ubicados en el peri-domicilio (Jutiapa $=76.3 \%$, Jarabacoa $=93.7 \%$ ) en depósitos permanentes y útiles (Jutiapa $=79.6$ $\%$, Jarabacoa $=56.9 \%)$. De forma general, Ae. aegypti fue la especie que más depósitos colonizó (Jutiapa $=92$, Jarabacoa $=84$ ), en cohabitación o no con otras especies. Los resultados refuerzan la necesidad de desarrollar estrategias de comunicación y participación comunitaria contextualizadas a las características de cada territorio y especie, así como direccionar los esfuerzos hacia el control doméstico, teniendo en cuenta sus contribuciones a la efectividad y sostenibilidad del manejo integrado de culícidos en estos y otros municipios con características similares.

Palabras claves: entomología; Culicidae; vectores de enfermedades; prevención primaria; región del Caribe.

\section{Introducción}

Las enfermedades vectoriales representan el $17 \%$ dela carga mundial estimada para las enfermedades infecciosas con altos índices de mortalidad, morbilidad, incapacidades laborales y pérdidas económicas. ${ }^{1}$ En relación a los arbovirus, el número de especies de artrópodos con potencialidad para transmitir estos patógenos es elevado, pero destacan sobre todo aquellas que exhiben hábitos antropófilos y sinantrópicos, ${ }^{2}$ es decir, las que tienen especial querencia por alimentarse de la sangre de los seres humanos y que viven estrechamente vinculadas a sus asentamientos. Dos especies de mosquitos (Diptera: Culicidae) con estas características desempeñan un papel protagónico en la región de las Américas: Aedes aegypti (Linnaeus, 1774) y Aedes albopictus (Skuse, 1894); vectores, entre otras enfermedades, del dengue, chikungunya y Zika. ${ }^{3}$ Por su parte, Culex quinquefasciatus Say, 1823 es un vector importante de encefalitis zoonóticas de origen viral, así como del parásito Wuchereria bancrofti (Cobbold, 1877) (Spirurida: Onchocercidae), agente causal de la filariosis linfática. ${ }^{4}$ both included in the LMIC classification. In each municipality, 100 randomly selected households were sampled; in each breeding site, as many larvae and/or pupae as possible were captured with the help of Pasteur pipettes, plastic trays and hermetic jars. The percentage of water holding containers infested by any of the Culicidae species studied was similar in both municipalities (Jutiapa $=27.9 \%$, Jarabacoa $=20.0 \%$ ); among these, those located in the peri-domicile (Jutiapa $=76.3 \%$, Jarabacoa $=93.7 \%$ ) and in permanent and serviceable containers (Jutiapa $=79.6 \%$, Jarabacoa $=56.9 \%)$ predominated. In general, Ae. aegypti was the species that colonized the most deposits (Jutiapa $=92$, Jarabacoa $=84)$, whether or not in cohabitation with other species. The results reinforce the need to develop communication and community awareness strategies contextualized to the characteristics of each territory and species, as well as to focus efforts towards domestic control, taking into account their contributions to the effectiveness and sustainability of integrated management of culicids in these and other municipalities with similar characteristics.

Keywords: entomology; Culicidae; disease vectors; primary prevention; Caribbean region.

Las campañas de erradicación de Ae. aegypti, principal agente transmisor de arbovirus en las Américas, fueron muy exitosas durante la década de los 50 y 60. Dichos esfuerzos contribuyeron a la eliminación de este vector en muchos países de la región. Sin embargo, la falta de sostenibilidad y, en algunos casos, el abandono de dichos programas resultó a partir de la década de los 70 en la reinfestación de esta especie, fundamentalmente en los países de bajos y medianos ingresos (LMIC, por sus siglas en inglés); perdiéndose de este modo el esfuerzo realizado en los ańos anteriores. ${ }^{5}$ Desde la fecha, motivados por la reemergencia de enfermedades como el dengue y por aspectos operacionales asociados al control de su vector, comenzó a exhortarse a la incorporación de la comunidad en las acciones de vigilancia y prevención, proceso que pasó a desempeñar un componente clave dentro de las estrategias de control antivectorial. ${ }^{6,7}$

Desde inicios del presente siglo se han producido brotes epidémicos muy intensos y reiterados en diversos países tropicales de las Américas, ${ }^{8}$ con 
una clara tendencia al incremento en LMIC en los últimos ańos. ${ }^{9}$ Este escenario entomo-epidemiológico se ha tornado más complejo debido, entre otros factores, al establecimiento de nuevas especies de mosquitos invasores en el continente como Ae. albopictus en la década de los $80,{ }^{10} \mathrm{y}$ Aedes vittatus (Bigot, 1851) más recientemente; ${ }^{11,12}$ a la aparición de resistencias a diferentes productos biocidas; ${ }^{13}$ así como a la co-infección y co-circulación de diversas arbovirosis. ${ }^{14}$ Asimismo, es importante tener en cuenta que, a raíz de la pandemia de la COVID-19, cuyas futuras repercusiones son todavía hoy inciertas, se ha producido un debilitamiento de los sistemas de salud que gestionan, tratan y controlan los casos de enfermedades de transmisión vectorial, ${ }^{15}$ lo que supone un hándicap añadido.

Teniendo en cuenta las consideraciones antes expuestas, el objetivo del presente trabajo fue caracterizar la infestación de las tres especies de culícidos sinantrópicos de mayor relevancia vectorial (Ae. aegypti, Ae. albopictus y $C x$. quinquefasciatus) en viviendas de dos LMIC de la región del Caribe, así como proponer recomendaciones para su control doméstico contextualizadas a cada situación.

\section{Materiales y métodos}

El estudio se llevó a cabo en Guatemala y República Dominicana durante el último trimestre de 2017, teniendo en cuenta que en ambos países existen reportes previos de infestación de las especies de mosquitos incluidas en el estudio y de la incidencia de enfermedades trasmitidas por estos. Además, están incluidos entre los LMIC, uno perteneciente a Centro América y otro al Caribe. Posteriormente, se seleccionó un municipio de cada país, mediante un muestreo por conveniencia.

\section{- Descripción de las áreas de estudio}

- República de Guatemala, departamento de Jutiapa, municipio de Jutiapa. Guatemala es un país situado en el extremo noroccidental de América Central. El municipio de Jutiapa se ubica en la región IV o región sur-oriente, con un área de $620 \mathrm{~km}^{2}$ y una altitud media de 905 msnm. Este municipio guatemalteco tiene un clima tropical, considerado Aw según la clasificación climática de Köppen-Geiger, con una temperatura media anual de $22.3{ }^{\circ} \mathrm{C}$ y una precipitación de $1.146 \mathrm{~mm}$ al año.

- República Dominicana, provincia de La Vega, municipio de Jarabacoa. República Dominicana es un país situado en el Caribe, ubicado en la zona central de las Antillas y que ocupa la parte centro-oriental de la isla de la Hispaniola. El municipio de Jarabacoa se ubica en un valle intramontano de la Cordillera Central, con un área de $23 \mathrm{~km}^{2}$ y una altitud media de $530 \mathrm{msnm}$. Este municipio dominicano tiene un clima tropical, considerado Af según la clasificación climática de Köppen-Geiger, con una temperatura media anual de $20.2^{\circ} \mathrm{C}$ y una precipitación de $1.723 \mathrm{~mm}$ al año.

\section{- Trabajo de campo}

En cada municipio se muestrearon 100 viviendas seleccionadas aleatoriamente: la selección de las mismas se realizó de acuerdo a una numeración preestablecida de las manzanas de cada localidad, dando sus moradores la aprobación (consentimiento informado) para su inspección intra y peri-domiciliar, dirigida a localizar criaderos potenciales de culícidos. En cada criadero se capturó la mayor cantidad posible de larvas y/o pupas con la ayuda de pipetas Pasteur, bandejas plásticas y botes herméticos.

La clasificación de los criaderos se realizó atendiendo a la utilidad que le dan las familias a los mismos: a) como permanentes y útiles (PU) se clasificaron los recipientes artificiales que casi siempre acumulan agua para diversos usos cotidianos; b) como no permanentes (NP) los recipientes artificiales que 


\section{Lorenzo Diéguez Fernández, Milton Vicio Monzón Muñoz, Jaime Abraham Juárez Sandoval, Dex Yorman Barrios \\ Barrios, Mónica Elisa Barrientos Juárez, Yisel Hernández Barrios, María Altagracia Rodríguez-Sosa, Yohan Enmanuel Vásquez-Bautista y Pedro María Alarcón-Elbal}

no acumulan agua con frecuencia y que, generalmente, están abandonados en áreas exteriores de las viviendas; y c) como naturales $(\mathrm{N})$ aquellos que no tienen un origen antrópico, formando parte del medio ambiente. ${ }^{16}$

Todas las muestras se acompañaron de una etiqueta en la que se reflejó el tipo de criadero, la ubicación en la vivienda, la fecha de captura y las coordenadas geográficas.

\section{- Trabajo de laboratorio}

El material biológico capturado en Guatemala se identificó en el Laboratorio de Entomología del Departamento de Control de Vectores del Área de Salud de Jutiapa, y el de República Dominicana en el Laboratorio de Entomología de la Universidad Agroforestal Fernando Arturo de Meriño (UAFAM) de Jarabacoa.

En el insectario de cada laboratorio, las muestras se introdujeron en botes de eclosión para el desarrollo de los ejemplares hasta alcanzar el estadio larvario $\mathrm{L}_{4}$, tras lo que se procedió a su fijación en alcohol al $70 \%$, previa muerte por baño de agua caliente a $60{ }^{\circ} \mathrm{C}$. Las pupas se dejaron eclosionar para realizar la identificación de los adultos, los que se sacrificaron por congelación y se conservaron en seco. Para la identificación taxonómica se utilizaron los criterios de Clark-Gil y Darsie ${ }^{17}$ y González Broche. ${ }^{18}$

\section{- Análisis de los datos}

Se realizó un análisis descriptivo en el cual se calcularon los siguientes porcentajes:

- Porcentaje de representatividad de Viviendas Positivas a Culícidos (VPC):

Total de viviendas positivas

$\mathrm{VPC}=\frac{\text { a culícidos }}{\text { Total de viviendas inspeccionadas }} \times 100 \%$

- Porcentaje de representatividad de Viviendas Positivas a las Especies Estudiadas (VPEE):

Total de viviendas positivas a las

$\mathrm{VPEE}=\ldots$ especies estudiadas $\quad$ X $100 \%$

Total de viviendas positivas a culícidos

- Porcentaje de representatividad de Depósitos Positivos a Culícidos (DPC):

Total de depósitos positivos

$\mathrm{DPC}=\frac{\mathrm{a} \text { culícidos }}{\text { Total de depósitos inspeccionados }} \mathrm{X} 100 \%$

- Porcentaje de representatividad de los Depósitos Positivos a las Especies Estudiadas (DPEE):

Total de depósitos positivos a las

DPEE $=\ldots$ especies estudiadas $\quad$ X $100 \%$

Total de depósitos positivos a culícidos

- Porcentaje de representatividad según la Disposición de los Depósitos Positivos a las Especies Estudiadas (DDPEEI, DDPEEP):

Total de depósitos positivos a las especies
DDPEEI $=$ estudiadas intra-domicilio $\quad$ X $100 \%$
Total de depósitos positivos a las especies estudiadas

Total de depósitos positivos a las especies DDPEEP $=\stackrel{\text { estudiadas peri-domicilio }}{\text { X } 100 \%}$
Total de depósitos positivos a las especies estudiadas

Porcentaje de representatividad según la Utilidad de Depósitos Positivos a las Especies Estudiadas (UDPPU, UDPNP y UDPN):

Total de depósitos $\mathrm{PU}$ positivos a las

UDPPU $=\frac{\text { especies estudiadas }}{\begin{array}{c}\text { Total de depósitos positivos a las } \\ \text { especies estudiadas }\end{array}} \times 100 \%$


Total de depósitos NP positivos a las

$$
\begin{gathered}
\text { UDPNP }=\frac{\text { especies estudiadas }}{\begin{array}{c}
\text { Total de depósitos positivos a las } \\
\text { especies estudiadas }
\end{array}} \times 100 \% \\
\text { UDPN }=\frac{\text { Total de depósitos N positivos a las }}{\text { especies estudiadas }} \text { Total de depósitos positivos a las } \\
\text { especies estudiadas }
\end{gathered}
$$

Para la representación de las cohabitaciones se utilizó el diagrama de Venn de tres conjuntos que muestra gráficamente las diferentes relaciones entre las especies estudiadas.

\section{- Clasificación de las acciones antivectoriales}

A tenor de los resultados encontrados a nivel entomológico, se plasman una serie de recomendaciones para el control doméstico de las especies de estudio, contextualizadas para cada uno de los dos municipios y clasificadas según $\mathrm{DNHE}^{19}$ en:

- Modificaciones Ambientales (MoA): formas de ordenamiento del medio consistentes en cualquier actividad periódica planificada, dirigida a originar condiciones permanentes desfavorables para la cría de vectores en sus hábitats.

- Manipulaciones Ambientales (MaA): formas de ordenamiento del medio consistentes en cualquier actividad periódica planificada, dirigida a originar condiciones temporales desfavorables para la cría de vectores en sus hábitats.

\section{Resultados}

En Jutiapa, en el $66 \%$ de las viviendas muestreadas se encontraron larvas y/o pupas de mosquitos, detectándose la presencia de al menos una de las tres especies objeto de estudio en 60 (90.9\%) (figura 1A). En total, se muestrearon 398 depósitos con agua, de los que 109 (27.9\%) fueron positivos a culícidos, mientras que en 93 depósitos (85.3\%) se detectó la presencia de al menos una de las tres especies (figura 1B). De entre estos últimos, la mayoría se ubicaron en el peri-domicilio $(n=71$, $76.3 \%$ ) (figura 1C) y correspondieron a depósitos PU (n = 74, $79.6 \%$ ) (figura 1D). Respecto a las cohabitaciones, Ae. aegypti y Ae. albopictus aparecieron cohabitando el mismo recipiente en nueve ocasiones, mientras que Ae. aegypti y $C x$. quinquefasciatus se capturaron juntos cuatro veces (figura 1E). Durante el muestreo se encontraron otras especies, en cohabitación o no con las del objeto de estudio, siendo estas: Aedes atropalpus (Coquillet, 1902), Culex coronator Dyar y Knab, 1906 y Psorophora confinnis (Lynch Arribálzaga, 1891).

Por su parte, en Jarabacoa el $77 \%$ de las viviendas encuestadas fueron positivas a mosquitos, detectándose la presencia de al menos una de las tres especies objeto de estudio en 74 (96.1\%) (figura 2A). Se muestrearon un total de 419 depósitos, de los que $84(20.1 \%)$ fueron positivos a la presencia de larvas y/o pupas de al menos una de las tres especies (figura 2B). De entre estos últimos, la mayoría se ubicaron en el peri-domicilio ( $\mathrm{n}=74,93.7 \%$ ) (figura 2C) y correspondieron a depósitos PU ( $\mathrm{n}=45,56.9 \%)$ (figura 2D). Aedes aegypti y Ae. albopictus aparecieron cohabitando el mismo recipiente en 30 ocasiones, mientras que las tres especies se capturaron juntas 12 veces (figura $2 \mathrm{E}$ ). Durante el muestreo se hallaron otras especies, en cohabitación o no con los tres culícidos que fueron objeto de estudio, siendo estas: Aedes albonotatus (Coquillett, 1905), Culex corniger Theobald, 1903, Culex janitor Theobald, 1903, Culex nigripalpus Theobald, 1901, Culex secutor Theobald, 1901, Toxorhynchites portoricensis (Von Röder, 1885) y Wyeomyia mitchellii (Theobald, 1905).

De forma general, Ae. aegypti fue la especie que más depósitos colonizó con un total de 176 positivos (92 en Jutiapa y 84 en Jarabacoa), en cohabitación o no con otras especies, seguida de Ae. albopictus con 41 depósitos (13 en Jutiapa y 28 en Jarabacoa) $y$, finalmente, Cx. quinquefasciatus con 26 (8 en Jutiapa y 18 en Jarabacoa) (figuras 1E y 2E). 


\section{Lorenzo Diéguez Fernández, Milton Vicio Monzón Muñoz, Jaime Abraham Juárez Sandoval, Dex Yorman Barrios \\ Barrios, Mónica Elisa Barrientos Juárez, Yisel Hernández Barrios, María Altagracia Rodríguez-Sosa, Yohan Enmanuel Vásquez-Bautista y Pedro María Alarcón-Elbal}

En las tablas 1 y 2 se muestran las acciones que las familias pueden aplicar según el tipo de depósito, para evitar la presencia de mosquitos en Jutiapa y Jarabacoa, respectivamente. Esta propuesta se originó a partir de la práctica acumulada por la red de control de vectores de ambos países, y teniendo en cuenta la factibilidad y accesibilidad para su aplicación por parte de las familias jutiapanecas y jarabacoenses, así como la experiencia acopiada por ambos equipos de investigación, respetando la cultura, conocimientos $\mathrm{y}$ costumbres de ambas poblaciones.

De las diez acciones propuestas, nueve son físicas y una es biológica. En este sentido, dentro de las acciones MoA hubo un comportamiento similar entre ambos municipios, pues las mismas en los depósitos PU se pueden aplicar en el $100 \%$ de los depósitos con presencia de larvas (9 tipos en Jutiapa y 10 tipos en Jarabacoa). Mientras, en los depósitos NP en Jutiapa se pueden aplicar en el $55.5 \%$ y en Jarabacoa en el $60.0 \%$. Respecto a la aplicación de peces de probada efectividad como biorreguladores, el total de tipos de depósitos PU en los que se puede usar este tipo de control en Jutiapa resultó ser superior al de Jarabacoa, al reportar el municipio guatemalteco una mayor positividad $(54.5 \%$ vs. $11.0 \%)$.

En ambos municipios se destaca la posibilidad de reutilizar los depósitos positivos, sin que implique afectaciones al ambiente. En Jutiapa, esta alternativa es posible en el $47.4 \%$ del total de depósitos $(\mathrm{n}=19)$, mientras que en Jarabacoa lo es en el $55.2 \%(\mathrm{n}=29)$.

\section{Discusión}

Guatemala y República Dominicana presentan características epidemiológicas y ambientales proclives para la circulación de enfermedades transmitidas por $A$ e. aegypti, Ae. albopictus y $C x$. quinquefasciatus, debido a factores como la mala urbanización, el calentamiento global, la pobreza y el acceso a la educación que, entre otras, colaboran sinérgicamente para que estos males persistan en los países. ${ }^{2,20}$
En Jutiapa, se producen procesos elevados de desertificación, sequías y alteración de ciclo de lluvias como consecuencia del deterioro del medio ambiente, la deforestación y contaminación de las cuencas hídricas, por lo que una práctica común en sus pobladores es la tenencia o presencia de numerosos depósitos artificiales que favorecen la acumulación de agua, dentro y en los alrededores de las viviendas, lo que contribuye a la presencia de varias especies de mosquitos. ${ }^{21}$ Con relación al agua potable, su disponibilidad en cantidad y calidad constituye un aspecto inseparable del saneamiento y control de estas especies. Esto es debido, fundamentalmente, a que la proliferación de mosquitos se produce por la acumulación de depósitos que pueden contener agua, tanto aquellos utilizados para el almacenamiento del líquido en situaciones de déficit en los hogares, como los residuos sólidos urbanos que se acumulan en los espacios públicos y privados; ${ }^{22}$ tangencialmente, también influyen otros factores como la falta de ordenamiento ambiental y las deficiencias del alcantarillado pluvial. ${ }^{23}$

A lo antes expuesto, se suman las limitaciones en cuanto a la movilización de recursos humanos y financieros para garantizar el control sostenible y eficiente de estas especies, lo que constituye un desafío adicional para LMIC de Centro América y el Caribe. De este modo, aportar evidencias sobre la infestación general de estas especies en el ámbito domiciliar (positividad y ubicación de cada depósito por especies, tipos de depósitos en los que estas cohabitan, y características de los mismos, entre otros) puede contribuir a redirigir las acciones actuales de prevención y control de las enfermedades transmitidas por las especies de culícidos estudiadas, tanto en estas como en otras áreas con características similares.

Con relación al dengue, una de las enfermedades con mayor incidencia en la región, Guatemala, presentó durante 2018 un incremento del $77 \%$ de los casos comparado con el ańo anterior, mientras que en 2019 se incrementó seis veces el número de casos 
con respecto a 2018. En tanto, en el departamento de Jutiapa se registró la quinta incidencia acumulada (536.0 por 100,000 habitantes) más elevada del país en 2019. ${ }^{24}$ En República Dominicana también se observó un gran incremento en el número de casos de dengue en 2019, arrojando una tasa de incidencia más alta, aunque una letalidad más baja en comparación con el resto de los años: particularmente en La Vega, provincia donde se ubica el municipio de Jarabacoa, se registró una de las incidencias acumuladas (43.7 por 100,000 habitantes) más bajas del país en $2019 .{ }^{25}$

Sin embargo, a pesar de la incidencia de esta arbovirosis en ambos países; así como de otras enfermedades transmitidas por especies eminentemente sinantrópicas, las publicaciones científicas que profundizan acerca de los depósitos más frecuentes en el entorno doméstico continúan siendo escasas y puntuales, dificultando el desarrollo de acciones domiciliares de prevención y control, contextualizadas y sustentadas en la evidencia científica. De hecho, este manuscrito complementa a algunas publicaciones anteriores que trataron el tema desde un enfoque menos amplio, haciendo referencia a la situación particular de alguna de estas especies en Jutiapa y Jarabacoa, ${ }^{26,27,28,29}$ adoptando un cariz más integral al comparar la situación entre ambos países y estudiar la complejidad de las interacciones de las especies sinantrópicas en el mismo contexto; además, trasciende del plano meramente entomológico al identificar una serie de recomendaciones de control domésticas, involucrando así a la responsabilidad ciudadana, adaptadas a la situación específica de cada municipio.

De forma general, el porcentaje de viviendas infestadas por mosquitos fue mayor en Jarabacoa (77 \%) que en Jutiapa (66 \%) (figuras 1A y 2A). Por otro lado, el porcentaje de depósitos con agua infestados por alguna de las especies de culícidos estudiados fue similar en ambos municipios, aunque mayor en Jutiapa (27.9\%) que en Jarabacoa (20.0 \%) (figuras $1 \mathrm{~B}$ y $2 \mathrm{~B})$. Los resultados obtenidos para este muni- cipio dominicano coinciden, además, con los de un estudio reciente sobre los recipientes colonizados por mosquitos sinantrópicos acometido en nueve provincias del país (19.6\%), el primero de este tipo en abarcar las tres macro-regiones de República Dominicana. ${ }^{30}$

Existe una preponderancia de los recipientes infestados por al menos una de las tres especies de importancia sanitaria en el peri-domicilio con respecto a los ubicados en el interior de las casas, siendo este predominio mayor en el municipio de Jarabacoa $(93.7 \%)$ que en Jutiapa (76.3 \%) (figuras 1C y 2C). Esto es debido a que suele haber una mayor disponibilidad de lugares para oviponer en los alrededores de las viviendas, no solo los dedicados a los diferentes usos domésticos (por ejemplo, tanques, tinacos y bebederos), sino también los que se encuentran en los patios y jardines, y que no tienen una utilidad definida (por ejemplo, neumáticos usados, botellas y botes). En este sentido, estudios realizados en Cuba demostraron que Ae. aegypti y $C x$. quinquefasciatus fueron las especies más numerosas al colonizar una gran variedad de recipientes inspeccionados, al no alejarse de sus criaderos y presentar fundamentalmente hábitos endofílicos; ${ }^{31}$ mientras, Marquetti et al. ${ }^{32}$ encontraron que los neumáticos usados, latas y bebederos fueron los depósitos que aportaron una importante presencia de estas dos especies de mosquitos. Asimismo, los reservorios naturales como las plantas, sobre todo las bromelias, propicias para el desarrollo de algunos aedinos, se encuentran de manera habitual en el exterior. De las tres especies de mosquitos estudiadas, Ae. aegypti es la que tiene un comportamiento antropófilo y endófilo más marcado. ${ }^{33}$ Sin embargo, suele encontrarse mayormente en recipientes ubicados al aire libre porque los moradores en general no se preocupan por la limpieza de estos contenedores, los cuales se llenan de agua durante las temporadas de lluvia. ${ }^{30,34}$

En cuanto a la finalidad de los contenedores, nuestros resultados mostraron que la presencia de al 


\section{Lorenzo Diéguez Fernández, Milton Vicio Monzón Muñoz, Jaime Abraham Juárez Sandoval, Dex Yorman Barrios \\ Barrios, Mónica Elisa Barrientos Juárez, Yisel Hernández Barrios, María Altagracia Rodríguez-Sosa, Yohan Enmanuel Vásquez-Bautista y Pedro María Alarcón-Elbal}

menos una de las tres especies de relevancia médica fue mayor en los depósitos PU en ambos municipios, siendo más acentuada en Jutiapa (79.6 \%) que en Jarabacoa (56.9\%) (figuras 1D y 2D). Esto puede explicarse por el hecho de que los contenedores PU almacenan agua de forma permanente para su uso esencial en las tareas domésticas, mientras que los NP retienen agua temporalmente, dependiendo, sobre todo, del régimen de lluvias. Esto hace que los primeros sean accesibles durante más tiempo que los segundos, siempre que no se tomen las medidas preventivas adecuadas. ${ }^{32}$

Aedes aegypti y Ae. albopictus presentaron los mayores valores absolutos en términos de cohabitación, tanto en Jutiapa como en Jarabacoa (figuras 1E y 2E), lo que coincide con otros estudios similares recientes realizados en las Américas. ${ }^{35} \mathrm{La}$ cohabitación de estas tres especies en recipientes domésticos ha sido descrita antes en países del Caribe como Cuba y República Dominicana. ${ }^{36,37}$ Estos tres culícidos sinantrópicos son oportunistas, razón por lo que poseen la capacidad de desarrollarse en una amplia variedad de criaderos artificiales del entorno doméstico, e incluso cohabitar de forma frecuente en los mismos, como se desprende del presente estudio. Sin embargo, a pesar de que $C x$. quinquefasciatus coloniza el espacio doméstico, no se encuentra tan abundantemente como los otros dos vectores. Al respecto, se ha evidenciado que los dos aedinos son capaces de colonizar criaderos artificiales de pequeńo y mediano tamaño de manera más eficiente, ${ }^{38}$ mientras que $C x$. quinquefasciatus también se encuentra fuera del ámbito doméstico con frecuencia, en criaderos naturales como charcos de agua de lluvia, lagunas, canales o zanjas, ${ }^{27}$ con una variación considerable tanto en las dimensiones del cuerpo de agua como del nivel de eutrofización del mismo. ${ }^{4}$

Cabe destacar que, por ser el peri-domicilio donde menos intervienen las familias para garantizar un adecuado saneamiento ambiental, es aquí donde hubo una mayor disponibilidad de depósitos para los mosquitos. Sin embargo, la posibilidad de reutilizar el $75 \%$ de los tipos de depósitos que resultaron positivos a culícidos, coloca al control antivectorial en un importante marco de gestión ambiental con alcance incluso hacia otras especies de vectores. Con esa reutilización se estaría contribuyendo a bajar sensiblemente la carga ambiental de estos depósitos, sobre todo los NP, entre los que destacan los neumáticos usados. Estos residuos sólidos, generalmente almacenados cerca de las poblaciones humanas en tiendas de venta y reparación de neumáticos, pero también habituales dentro de las casas, representan una grave amenaza para el medio ambiente y la salud pública, pues tienen el potencial de albergar una gran diversidad de mosquitos vectores de enfermedades. ${ }^{39}$ Sin embargo, existen alternativas económicas y ecológicas notables, como el reciclaje de estos neumáticos en macetas, elementos recreativos y mobiliario, entre otros (figura 3). A nivel de comunidad, medidas como las anteriores y otras como la reducción sistemática de desechos en el entorno doméstico, conocido en muchos países como «descacharrización», la procuración de la hermeticidad de los depósitos destinados a la acumulación de agua para uso doméstico, y la desecación de lugares proclives a inundarse son esenciales para la reducción y el control de criaderos potenciales de mosquitos en las casas. Aunque en algunas ocasiones también se procede a la quema a cielo abierto de desechos que pueden ser criaderos potenciales, lo cierto es que esta actividad comporta un grave riesgo para la salud humana, ya que se produce una enorme carga de dioxinas, sobre todo cuando son residuos plásticos. Por tanto, esta alternativa solo es aconsejable con criaderos potenciales de origen orgánico, como jícaras de coco o yaguas de ciertas especies de palmas.

Las iniciativas contemporáneas favorecen la participación comunitaria, el manejo ambiental y la comunicación social como componentes claves para el 
manejo integrado de vectores y las enfermedades transmisibles. ${ }^{40}$ No obstante, en las últimas décadas los esfuerzos llevados a cabo sobre el desarrollo y la articulación de esos componentes con el resto de las acciones de los programas de control resultan insuficientes en ambos municipios estudiados. Además, entre los moradores predomina la tendencia a desplazar la responsabilidad y carga socioeconómica de las acciones de prevención, principalmente, a los sectores y decisores involucrados en el control del ambiente y la salud. De forma conjunta, se percibe un incremento, entre otros aspectos, de la urbanización, la densidad y movilidad poblacional, y los patrones conductuales (por ejemplo, acumulación de agua en una amplia variedad de depósitos, poca sistematicidad en su revisión, ausencia o deficiencias en la aplicación de productos biocidas) que favorecen la proliferación de mosquitos de importancia médica y, en consecuencia, el riesgo de transmisión de enfermedades asociadas a los mismos.

En sentido general, la mayoría de los intentos por incorporar a la comunidad de manera activa y consciente a los programas de vigilancia y control antivectorial han fracasado. ${ }^{41} \mathrm{~A}$ saber, una de las razones de este fracaso es que la población no ha participado ni en su diseño estratégico ni en el establecimiento de sus objetivos, y mucho menos en la toma de decisiones, lo que se agrava al no contar con suficiente apoyo gubernamental, hecho que coincide con los resultados hallados desde finales del siglo pasado por Service ${ }^{42}$ y Rifkin. ${ }^{43}$ Sin embargo, la implementación reciente de la iniciativa local "Sácale los pies al mosquito», llevada a cabo en Jarabacoa, ha demostrado que se pueden conseguir buenos resultados si se involucra a la población desde las primeras etapas de los programas, sobre todo a los jóvenes en edad escolar. ${ }^{44}$

Asimismo, la diversidad de depósitos positivos y las diferencias en cuanto a la infestación de las tres especies por áreas de estudio refuerzan la importancia de los elementos contextuales y sugieren la necesidad de, sin descuidar aspectos comunes de la ecología de estos vectores y los programas regionales afines, contextualizar las acciones de control a las particularidades entomológicas y sociales de cada municipio y país. También es necesario desarrollar acciones de comunicación y participación comunitaria que contribuyan a involucrar a las comunidades en el control de estas especies, y optimizar los recursos en estos y otros municipios que pertenezcan a LMIC, para garantizar la efectividad y sostenibilidad de las acciones. Las acciones antivectoriales propuestas para cada municipio (tablas 1 y 2 ) resultan ser de fácil implementación y no generan gastos económicos adicionales a las familias; además, con ello se garantizaría la creación de condiciones favorables para el control racional de las especies de mosquitos sinantrópicas, que son el objeto del presente estudio.

Por último, es importante adoptar un enfoque interdisciplinario para minimizar los daños y maximizar los beneficios de la gestión conjunta de las personas, los animales y la salud ambiental, buscando desarrollar estrategias más eficientes y eficaces para hacer frente a los problemas de salud del trinomio ser humano-animal-medio ambiente..$^{45} \mathrm{Al}$ respecto, la entomología "One Health» debe jugar un papel importante en la primera línea del control de enfermedades vectoriales, siendo esta disciplina en particular importante en regiones que, como el Caribe, están continuamente amenazadas por el impacto de artrópodos perjudiciales debido a su clima y medio ambiente tropical, y agravado por sus limitados recursos económicos. ${ }^{15}$

\section{Conflicto de interés}

Todos los autores que firman este manuscrito han ameritado con trabajo, esfuerzo y dedicación su inclusión en el mismo; y manifiestan la no existencia de conflicto de interés. 


\section{Lorenzo Diéguez Fernández, Milton Vicio Monzón Muñoz, Jaime Abraham Juárez Sandoval, Dex Yorman Barrios \\ Barrios, Mónica Elisa Barrientos Juárez, Yisel Hernández Barrios, María Altagracia Rodríguez-Sosa, Yohan Enmanuel Vásquez-Bautista y Pedro María Alarcón-Elbal}

\section{Agradecimientos}

Los autores expresan su agradecimiento a los directivos del Área de Salud de Jutiapa, al Coordinador del Programa de Enfermedades Transmitidas por Vectores de Jutiapa, Sr. David Jiménez Gudiel, así como a los operativos y cadena de mando del Departamento de Control de Vectores de Jutiapa, por su apoyo incondicional a la parte de esta investigación desarrollada en Guatemala. Además, agradecemos encarecidamente a todos aquellos ciudadanos de Jutiapa y de Jarabacoa que nos permitieron acceder a sus hogares, prestando su colaboración de forma totalmente desinteresada para poder realizar este trabajo.

\section{Referencias}

1. Organización Mundial de la Salud. Fiebre amarilla. Respuesta Mundial para el Control de Vectores 2017-2030. [Internet]. Ginebra; 2017 [citado 15 mar 2021]. Disponible en: https://www.who.int/malaria/areas/vector_ control/Draft-WHO-GVCR-2017-2030-esp. pdf?ua $=1$.

2. Alarcón-Elbal PM, Paulino-Ramírez R, DiéguezFernández L, Fimia-Duarte R, Guerrero KA, González M. Arbovirosis transmitidas por mosquitos (Diptera: Culicidae) en la República Dominicana: una revisión. The Biologist (Lima). 2017;15(1):193-219. Disponible en: http:// revistas.unfv.edu.pe/index.php/rtb/article/ view/155/147

3. Weaver SC, Reisen WK. Present and future arboviral threats. Antivir. Res. 2010;85:32845. Available from https://doi.org/10.1016/j. antiviral.2009.10.008

4. Sajal-Bhattacharya PB. The Southern House Mosquito, Culex quinquefasciatus: profile of a smart vector. J. Entomol. Zool. Stud. 2016;4(2):73-81. Available from https:/www.entomoljournal.com/ archives/2016/vol4issue2/PartB/4-2-32.pdf
5. Pan American Health Organization (PAHO). Dengue and dengue hemorrhagic fever in the Americas: guidelines for prevention and control. Scientific Publication $\mathrm{N}^{\circ}$ 548. Washington: PAHO; 1997.

6. Gubler DJ, Clark GG. Community involvement in the control of Aedes aegypti. Acta Trop. 1996;61:169-79. Available from https://doi. org/10.1016/0001-706X(95)00103-L

7. Gubler DJ. (1998). Resurgent vector-borne diseases as a global health problem. Emerg. Infect. Dis. 1998;4:442-50. Available from https://www. ncbi.nlm.nih.gov/pmc/articles/PMC2640300/ pdf/9716967.pdf

8. San Martín JL, Brathwaite-Dick O. La Estrategia de Gestión Integrada para la prevención y el control del dengue en la región de las Américas. Rev. Panam. Salud Publica. 2007;21(1):55-63. Disponible en: https://scielosp.org/pdf/rpsp/ 2007.v21n1/55-63/es

9. Mathieu K, Karmali M. Vector-borne diseases, climatechangeandhealthyurbanliving:Nextsteps. Can. Commun. Dis. Rep. 2016;42(10):219-21. Available from https://www.ncbi.nlm.nih.gov/ pmc/articles/PMC5757676/pdf/CCDR-42219.pdf

10. Sprenger D, Wuithiranyagool T. The discovery and distribution of Aedes albopictus in Harris County, Texas. J. Am. Mosq. Control Assoc. $1986 ; 2: 217-9$.

11. Alarcón-Elbal PM, Rodríguez-Sosa MA, Newman BC, Sutton WB. The First Record of Aedes vittatus (Diptera: Culicidae) in the Dominican Republic: Public Health Implications of a Potential Invasive Mosquito Species in the Americas. J. Med. Entomol. 2020;57(6):2016-21. Available from https://doi.org/10.1093/jme/tjaa128 
12. Díaz Martínez I, Diéguez Fernández L, Santana Águila B, Atiénzar de la Paz EM, Ruiz Domínguez D, Alarcón-Elbal PM. New introduction of Aedes vittatus (Diptera: Culicidae) into the East-Central region of Cuba: ecological characterization and medical relevance. Interam. J. Med. Health. 2021;4. Available from https://www.iajmh.com/iajmh/article/ view/175/223

13. Rodríguez MM, Bisset JA, Fernández D. Levels of insecticide resistance and resistance mechanisms in Aedes aegypti from some Latin American countries. J. Am. Mosq. Control Assoc. 2007;23:420-9. Available from https://doi.org/10.2987/5588.1

14. Rodriguez-Morales AJ, Villamil-Gómez WE, Franco-Paredes $\mathrm{C}$. The arboviral burden of disease caused by co-circulation and co-infection of dengue, chikungunya and Zika in the Americas. Travel Med. Infect. Dis. 2016;14(3):177-9. Available from https://doi.org/10.1016/j.tmaid. 2016.05.004

15. Alarcón-Elbal PM, Sandiford SL. One Health entomology in the insular Caribbean: time to bet on prevention. Interam. J. Med. Health. 2021;4. Available from https://www.iajmh.com/ iajmh/article/view/170/232

16. Diéguez L, Cabrera SM, Prada Y, Cruz C, Rodríguez RA. Aedes (St.) aegypti en tanques bajos y sus implicaciones para el control del dengue en Camagüey. Rev. Cubana Med. Trop. 2010;62(2):93-7. Disponible en: http://scielo. sld.cu/pdf/mtr/v62n2/mtr02210.pdf

17. Clark-Gril S, Darsie RI. The mosquitoes of Guatemala. Their identification, distribution and bionomics. Mosq. Syst. 1983;15:1-231. Available from http://mosquito-taxonomic-inventory.info/sites/mosquito-taxonomic-inventory.info/files/Clark-Gil\%20\%26\%20 Darsie\%201983.pdf
18. González Broche R. Culícidos de Cuba. Cuba: Ed. Científico Técnica; 2006.

19. Dirección Nacional de Higiene y Epidemiología (DNHE). Higiene del Medio. Tomo I. Cuba: Ed. Pueblo y Educación; 1981

20. Organización Panamericana de la Salud. El dengue, chikungunya y Zika se pueden prevenir. Washington D.C.; OPS; 2021 [citado 15 mar 2021]. Disponible en: https://www.paho.org/ gut/index.php?option=com_content $\&$ view=article\&id=817:e-dengue-chikungunya-y-zika-sepueden-prevenir\&Itemid $=441$

21. Municipio de Jutiapa. Diagnóstico socioeconómico, potencialidades productivas y propuestas de inversión. Informe General. Guatemala: Universidad de San Carlos de Guatemala; 2013.

22. Borge de Prada M, Rodríguez-Sosa MA, VásquezBautista YE, Guerrero KA, Alarcón-Elbal PM. Mosquitos (Diptera, Culicidae) de importancia médica asociados a residuos sólidos urbanos en Jarabacoa, República Dominicana. Rev. Salud Jalisco. 2018;Edición Especial:20-27. Disponible en: https://www.medigraphic.com/pdfs/saljalisco/sj-2018/sj18Ed.pdf

23. Instituto Nacional de Aguas Potables y Alcantarillados. 2016. Propuesta de Estrategia Nacional de Saneamiento. República Dominica na: INAPA.

24. Sánchez Has A. Análisis de situación de dengue, Guatemala 2019. Ciudad de Guatemala: 2019 [citado 15 mar 2021]. Disponible en: http:// epidemiologia.mspas.gob.gt/files/2021/salas-situacionales/arbovirosis/Man-ARB-2019.pdf

25. Servicio Nacional de Salud. Reporte de análisis de dengue en la red pública. Boletín especial. Santo Domingo: SNS; 2019. 
26. Monzón MV, Rodríguez J, Diéguez L, AlarcónElbal PM, San Martín JL. Hábitats de cría de Aedes aegypti (Diptera: Culicidae) en Jutiapa, Guatemala. Novit. Caribaea, 2019;14:111-20. Disponible en: https://novitatescaribaea.do/ index.php/novitates/article/view/203/189

27. Rodríguez Sosa MA, Diéguez Fernández L, Borge de Prada M, Vásquez Bautista YE, Alarcón-Elbal PM. Sitios de cría de Aedes albopictus (Skuse) (Diptera: Culicidae) en el entorno doméstico en Jarabacoa, República Dominicana. Rev. Chilena Entomol. 2019;45(3):403-10. Disponible en: https://www.biotaxa.org/rce/ article/view/56096/50108

28. Diéguez Fernández L, Borge de Prada M, RodríguezSosa MA, Vásquez BautistaYE,AlarcónElbal PM. Un acercamiento al conocimiento de los hábitats larvarios de Aedes (Stegomyia) aegypti (Diptera: Culicidae) en el entorno doméstico en Jarabacoa, República Dominicana. Rev. Cubana Med. Trop. 2020;71(3):e386. Disponible en: http://scielo.sld.cu/pdf/mtr/v71n3/1561-3054mtr-71-03-e386.pdf

29. Diéguez Fernández L, Rodríguez Sosa MA, Vásquez Bautista YE, Borge de Prada M, Alarcón-Elbal PM. Aportes a la bioecología de Culex quinquefasciatus (Diptera: Culicidae), vector de encefalitis, en Jarabacoa, República Dominicana. Folia Entomol. Mex. 2020;6(2):26-32. Disponible en: https:// www.socmexent.org/folia/revista/Num $\% 20$ 2020_2/FEMns\%206(2)\%2026-32.pdf

30. Alarcón-Elbal PM, Rodríguez Sosa MA, RuizMatuk C, Tapia L, Arredondo Abreu CA, Fernández González AA, et al. Breeding sites of synanthropic mosquitoes (Diptera: Culicidae) in Zika-affected areas of the Dominican Republic. J. Am. Mosq. Control Assoc. 2021;37(1):10-9. Available from https://meridian.allenpress.com/ jamca/article-pdf/37/1/10/2810832/i8756971x-37-1-10.pdf
31. Peraza I, Pérez M, Mendizábal ME, Valdés V, Leyva M, Marquetti, MC. Riqueza y distribución de especies de culícidos en la provincia La Habana, Cuba. Rev. Cubana Med. Trop. 2015;67(2):270-8. Disponible en: http://scielo. sld.cu/pdf/mtr/v67n2/mtr12215.pdf

32. Marquetti MC, Peraza I, Pérez M, Mendizábal ME, Valdés V, Leyva $M$, et al. Riqueza de mosquitos en la Habana: su importancia para fomentar la participación comunitaria en su control. Rev. Cubana Med. Trop. 2019;71(3):112. Disponible en: http://scielo.sld.cu/pdf/mtr/ v71n3/1561-3054-mtr-71-03-e409.pdf

33. Carrington LB, Simmons CP. Human to mosquito transmission of dengue viruses. Front. Immunol. 2014;5:290. Disponible en: https://www.ncbi. nlm.nih.gov/pmc/articles/PMC4060056/pdf/ fimmu-05-00290.pdf

34. Jansen CC, Beebe NW. The dengue vector Aedes aegypti: what comes next? Microbes Infect. 2010;12:272-9. Available from https://doi.org/ 10.1016/j.micinf.2009.12.011

35. Piovezan R, Oliveira Acorinthe JP, Teixeira de Souza JH, Visockas A, de Azevedo TS, Von Zuben CJ. Spatial distribution of Culicidae (Diptera) larvae, and its implications for Public Health, in five areas of the Atlantic Forest biome, State of São Paulo, Brazil. Rev. Bras. Entomol. 2017;61(2):123-35. Available from https://doi. org/10.1016/j.rbe.2016.12.007

36. Diéguez L, Pino R, Andrés J, Hernández A, Alarcón-Elbal PM, San Martín JL. Actualización de los hábitats larvarios de Aedes aegypti (Diptera: Culicidae) en Camagüey, Cuba. Rev. Biol. Trop. 2016;64(4):1487-93. Available from https:// www.redalyc.org/pdf/449/44947539010.pdf 
37. Rodríguez Sosa MA, Rueda J, Vásquez Bautista YE, Fimia-Duarte R, Borge de Prada M, Guerrero $\mathrm{KA}$, et al. Diversidad de mosquitos (Diptera: Culicidae) de Jarabacoa, República Dominicana. Graellsia. 2019;75(1):e084. Disponible en: http:// graellsia.revistas.csic.es/index.php/graellsia/ article/view/591/772

38. Santana-Martínez JC, Molina J, Dussán J. Asymmetrical competition between Aedes aegypti and Culex quinquefasciatus (Diptera: Culicidae) coexisting in breeding sites. Insects, 2017;8(4):pii:E111. Available from https:// www.mdpi.com/2075-4450/8/4/111/pdf

39. González MA, Rodríguez Sosa MA, Vásquez Bautista YE, Rosario EC, Durán Tiburcio JC, Alarcón-Elbal PM. A survey of tire-breeding mosquitoes (Diptera: Culicidae) in the Dominican Republic: considerations about a pressing issue. Biomedica, 2020;40(3):507-15. Available from http://www.scielo.org.co/pdf/bio/ v40n3/2590-7379-bio-40-03-507.pdf

40. Organización Panamericana de la Salud. Evaluación de las estrategias innovadoras para el control de Aedes aegypti: desafíos para su introducción y evaluación del impacto. Washington, D.C.: OPS; 2019 [citado 15 mar 2021]. Disponible en: https://www.paho.org/es/documentos/evaluacion-estrategias-innovadoras-para-control-aedes-aegypti-desafios-para-su

41. Diéguez L, Cruz C, Acao L. Aedes (St.) aegypti: relevancia entomoepidemiológica y estrategias para su control. Arch. Med. Camagüey. 2011;15(3):604-19. Disponible en: http://scielo. sld.cu/pdf/amc/v15n3/amc180311.pdf
42. Service MW. Community participation in vector-borne disease control. Ann. Trop. Med. Parasitol. 1993;87:223-34. Available from https://doi.org/10.1080/00034983.1993.1 1812760

43. Rifkin SB. Paradigms lost: toward a new understanding of community participation in health programmes. Acta Trop. 1996;61:79-92. Available from https://doi.org/10.1016/0001 $-706 \mathrm{X}(95) 00105-\mathrm{N}$

44. Vásquez Bautista YE, Hernández Barrios Y, Rodríguez Sosa MA, Rosario EC, Durán Tiburcio JC, Alarcón-Elbal PM. «Sácale los pies al mosquito»: resultados parciales de la implementación de un programa educativo en República Dominicana. Cienc. Soc. 2019;44(3):33-49. Disponible en: https://revistas.intec.edu.do/ index.php/ciso/article/view/1487/2078

45. Organización Panamericana de la Salud. Lanzado nuevo programa "Una sola salud" para capacitar y empoderar a los profesionales de los sectores de salud, agricultura y medio ambiente. Washington D.C.; OPS; 2021 [citado 15 mar 2021]. Disponible en: https://www.paho.org/panaftosa/ index.php?option $=$ com_content $\&$ view $=$ article\&id=969: lanzado-nuevo-programa-una-sola-salud-para-capacitar-y-empoderar-a-profesionales-sectores-salud-agricultura-y-medio-ambiente \&Itemid = 504\#: : text $=\% 22 \mathrm{One} \%$ 20 Health \% E 2\%80\%9D\%20(Una\%20 Sola, an imales $\% 20$ y 201 a $\% 20$ salud \% 20ambiental. 
A

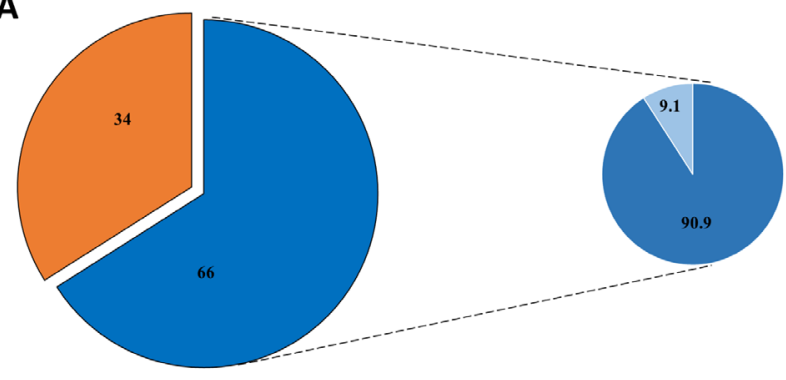

Viviendas negativa

Viviendas positivas

C

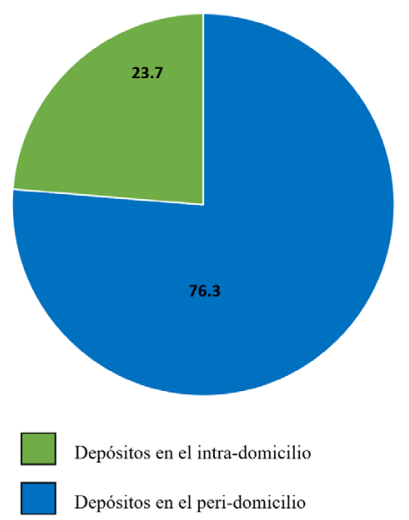

Viviendas positivas a las especies de mosquito estudiadas Viviendas positivas a otras especies de mosquitos
B

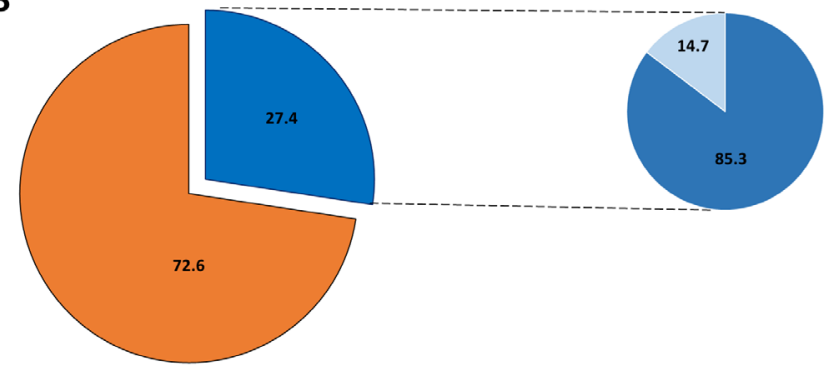

Depósitos negativos $\square$ Depósitos positivos a las especies de mosquito estudiadas Depósitos positivos $\square$ Depósitos positivos a otras especies de mosquitos
D

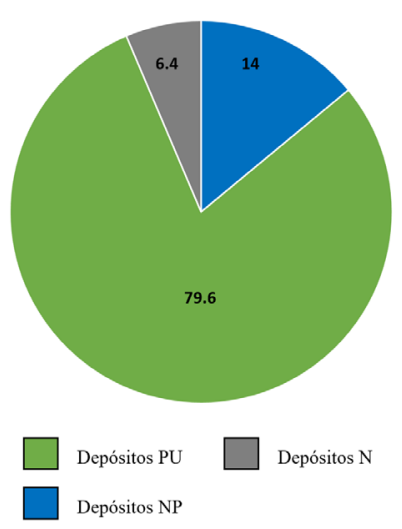

$\mathbf{E}$

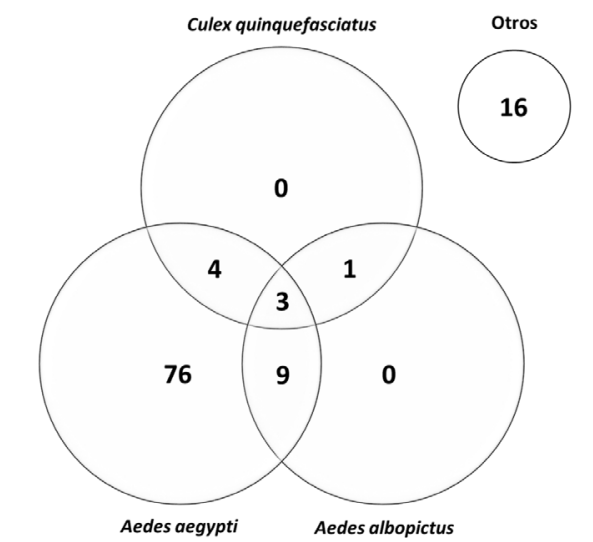

Figura 1. Situación entomológica sobre los culícidos sinantrópicos en Jutiapa, Guatemala, donde: A) Porcentaje de representatividad de viviendas positivas a culícidos y, de estas, porcentaje de viviendas positivas a las especies estudiadas; B) Porcentaje de representatividad de depósitos positivos a culícidos y, de estos, porcentaje de los depósitos positivos a las especies estudiadas; C) Porcentaje de representatividad según la disposición de los depósitos positivos a las especies estudiadas; D) Porcentaje de representatividad según la utilidad de los depósitos positivos a las especies estudiadas; y, E) Cohabitaciones entre las especies estudiadas.

Fuente: elaboración propia. 
A

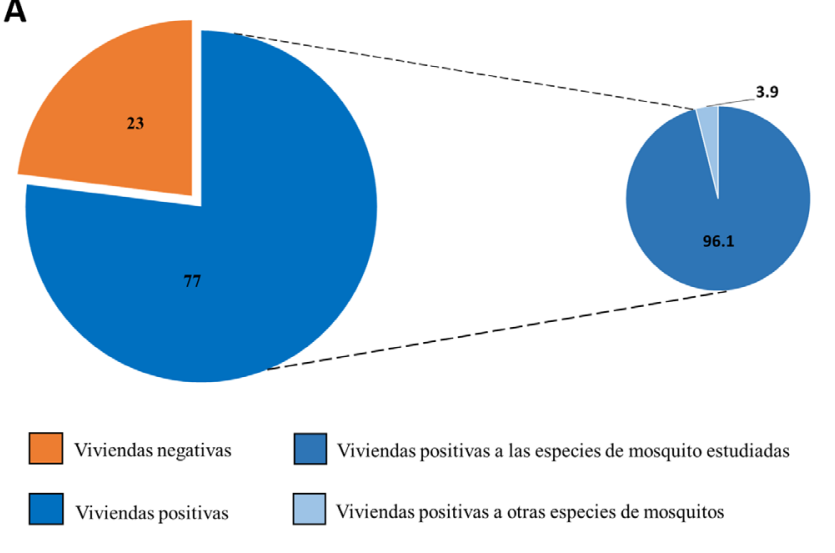

c

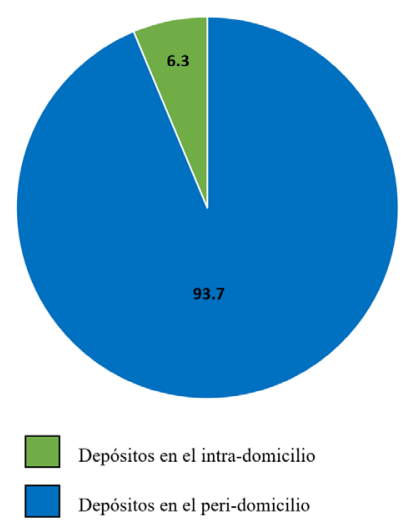

D

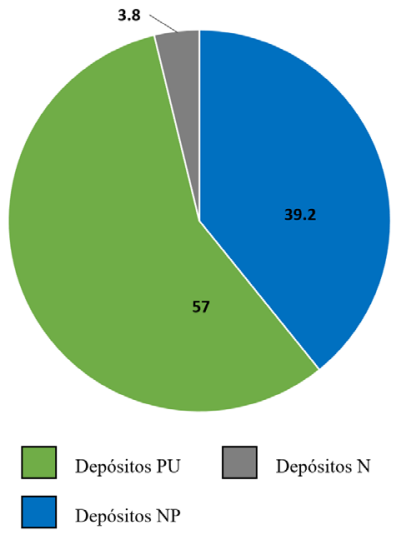

B

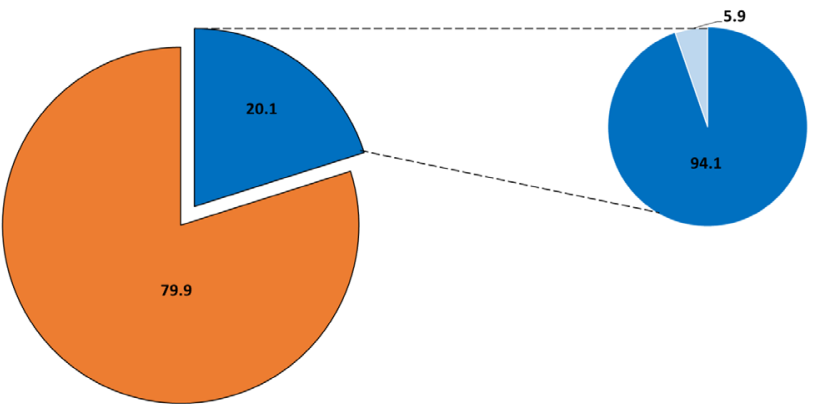

$\square$ Depósitos negativos $\square$ Depósitos positivos a las especies de mosquito estudiadas

Depósitos positivos $\square$ Depósitos positivos a otras especies de mosquitos

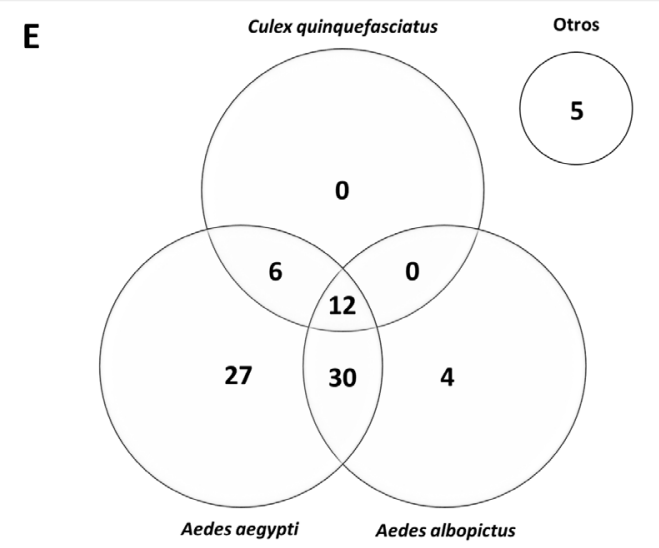

Figura 2. Situación entomológica sobre los culícidos sinantrópicos en Jarabacoa, República Dominicana, donde: A) Porcentaje de representatividad de viviendas positivas a culícidos y, de estas, porcentaje de viviendas positivas a las especies estudiadas; B) Porcentaje de representatividad de depósitos positivos a culícidos y, de estos, porcentaje de los depósitos positivos a las especies estudiadas; C) Porcentaje de representatividad según la disposición de los depósitos positivos a las especies estudiadas; D) Porcentaje de representatividad según la utilidad de los depósitos positivos a las especies estudiadas; y, E) Cohabitaciones entre las especies estudiadas.

Fuente: elaboración propia. 


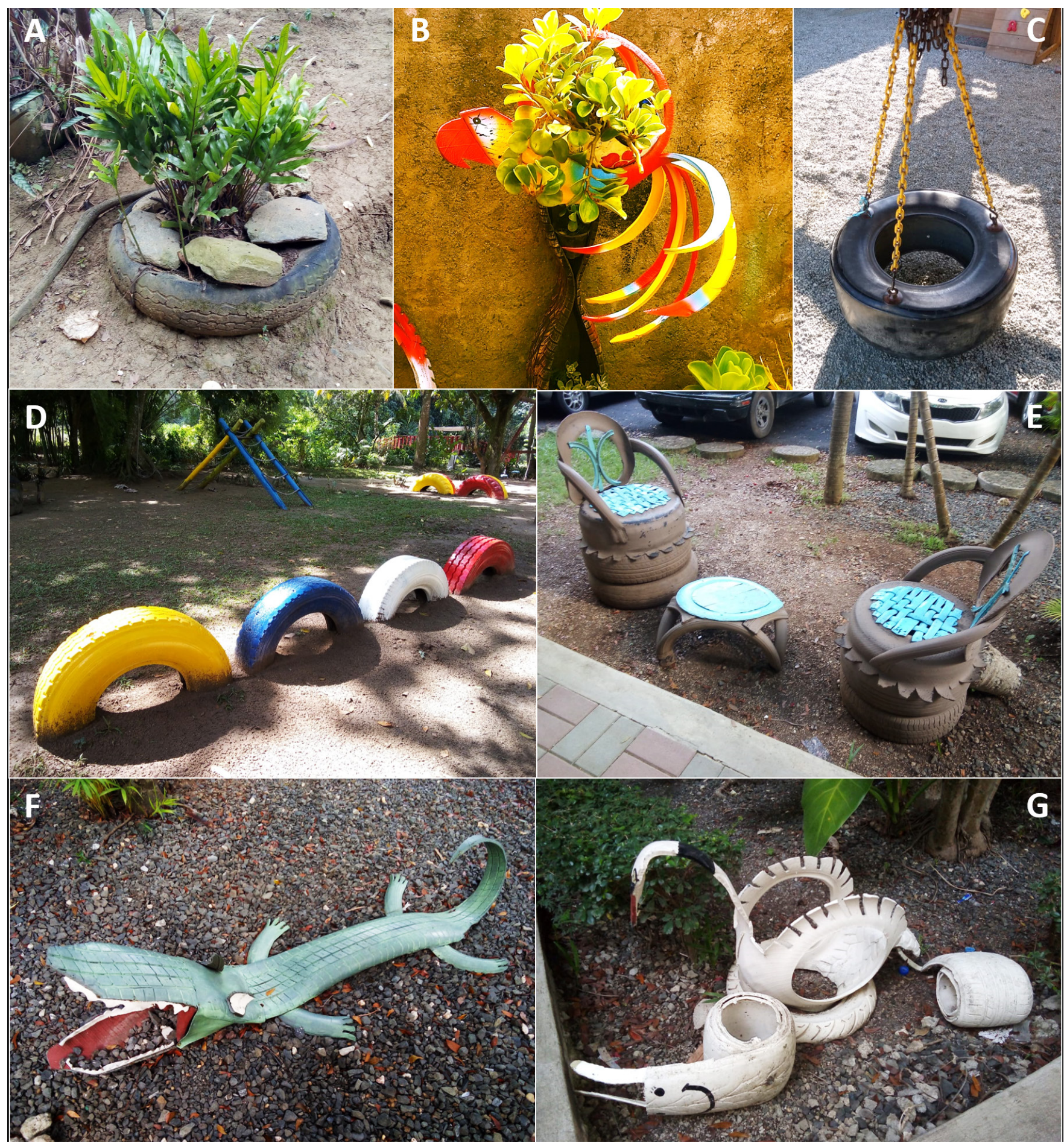

Figura 3. Diferentes usos alternativos de los neumáticos desechados en República Dominicana, donde: A) Arriate en sector Estancita, Jarabacoa; B) Macetero colgante en sector Pinar Quemado, Jarabacoa; C) Columpio en centro urbano, Jarabacoa; D) Parque infantil en La Trinchera, Jarabacoa; D) Mobiliario en la Universidad Central del Este (UCE), San Pedro de Macorís; F y G) Elementos decorativos en la UCE, San Pedro de Macorís.

Fuente: elaboración propia. 

médica en Países de Bajos y medios ingresos: recomendaciones para su control doméstico

Tabla 1. Acciones físicas y biológicas que las familias pueden aplicar en cada tipo de depósito para evitar la presencia de culícidos en Jutiapa, Guatemala

\begin{tabular}{|c|c|c|c|c|c|c|c|c|c|c|}
\hline \multirow[b]{3}{*}{ Depósitos } & \multicolumn{10}{|c|}{ Acciones a ejecutar por parte de las familias } \\
\hline & \multicolumn{8}{|c|}{ Físicas } & \multirow{2}{*}{$\begin{array}{c}\text { Biológicas } \\
\text { Aplicar } \\
\text { peces }\end{array}$} & \multirow[b]{2}{*}{$\begin{array}{c}\text { Total } \\
\text { acciones }\end{array}$} \\
\hline & Cepillar & Hermetizar & Flamear & $\begin{array}{c}\text { Piquetear } \\
\text { Aplastar }\end{array}$ & Virar & Quemar & $\begin{array}{c}\text { Colocar } \\
\text { bajo techo }\end{array}$ & Reutilizar & & \\
\hline \multicolumn{11}{|l|}{ Depósitos PU } \\
\hline Pila estándar & $\mathrm{X}$ & & $\mathrm{X}$ & & & & & & & 2 \\
\hline $\begin{array}{l}\text { Tonel } \\
\text { plástico }\end{array}$ & $\mathrm{X}$ & $\mathrm{X}$ & $\mathrm{X}$ & & $\mathrm{X}$ & & $\mathrm{X}$ & & $\mathrm{X}$ & 6 \\
\hline Tonel metal & $\mathrm{X}$ & $\mathrm{X}$ & $\mathrm{X}$ & & $\mathrm{X}$ & & $\mathrm{X}$ & & $\mathrm{X}$ & 6 \\
\hline $\begin{array}{l}\text { Tanque } \\
\text { plástico }\end{array}$ & $\mathrm{X}$ & $\mathrm{X}$ & $\mathrm{X}$ & & $\mathrm{X}$ & & $\mathrm{X}$ & & $\mathrm{X}$ & 6 \\
\hline $\begin{array}{l}\text { Tanque } \\
\text { metal }\end{array}$ & $\mathrm{X}$ & $\mathrm{X}$ & $\mathrm{X}$ & & $\mathrm{X}$ & & $\mathrm{X}$ & & $\mathrm{X}$ & 6 \\
\hline $\begin{array}{l}\text { Cubeta } \\
\text { plástica }\end{array}$ & $\mathrm{X}$ & $\mathrm{X}$ & $\mathrm{X}$ & & $\mathrm{X}$ & & $\mathrm{X}$ & $\mathrm{X}$ & & 6 \\
\hline Florero & $\mathrm{X}$ & & & & $\mathrm{X}$ & & $\mathrm{X}$ & $\mathrm{X}$ & & 4 \\
\hline Pozo & & $X$ & & & & & & & $X$ & 2 \\
\hline Palangana & $\mathrm{X}$ & & $\mathrm{X}$ & & $\mathrm{X}$ & & $\mathrm{X}$ & $\mathrm{X}$ & & 5 \\
\hline Caja de agua & & $\mathrm{X}$ & & & & & & & $X$ & 2 \\
\hline Total & 8 & 7 & 7 & & 7 & & 7 & 3 & 6 & 45 \\
\hline \multicolumn{11}{|l|}{ Depósitos NP } \\
\hline $\begin{array}{l}\text { Neumáticos } \\
\text { usados }\end{array}$ & & & $\mathrm{X}$ & $\mathrm{X}$ & & & $\mathrm{X}$ & $\mathrm{X}$ & & 3 \\
\hline Bote plástico & & & & $\mathrm{X}$ & & & & $\mathrm{X}$ & & 2 \\
\hline Lata & & & & $X$ & & & & $X$ & & 2 \\
\hline Olla metal & $\mathrm{X}$ & & $X$ & $X$ & $\mathrm{X}$ & & $\mathrm{X}$ & $X$ & & 6 \\
\hline $\begin{array}{l}\text { Bolsa } \\
\text { plástica }\end{array}$ & & & & & & & & & & 0 \\
\hline Sanitario & $\mathrm{X}$ & & & & & & $\mathrm{X}$ & $\mathrm{X}$ & & 3 \\
\hline $\begin{array}{l}\text { Jícara de } \\
\text { coco }\end{array}$ & & & & $\mathrm{X}$ & & $\mathrm{X}$ & & $\mathrm{X}$ & & 3 \\
\hline $\begin{array}{l}\text { Maceta de } \\
\text { cerámica }\end{array}$ & \multirow{2}{*}{\multicolumn{10}{|c|}{$\begin{array}{l}\text { Revisión periódica que no exceda los siete días, } \\
\text { sobre todo en el período con incremento de lluvias }\end{array}$}} \\
\hline Planta & & & & & & & & & & \\
\hline Total & 2 & & 2 & 4 & 1 & 1 & 3 & 6 & & 19 \\
\hline
\end{tabular}

Nota: en gris las acciones MoA en las que se crean condiciones permanentes desfavorables para la cría de vectores en sus hábitats.

Fuente: elaboración propia. 


\section{Lorenzo Diéguez Fernández, Milton Vicio Monzón Muñoz, Jaime Abraham Juárez Sandoval, Dex Yorman Barrios Barrios, Mónica Elisa Barrientos Juárez, Yisel Hernández Barrios, María Altagracia Rodríguez-Sosa, Yohan Enmanuel Vásquez-Bautista y Pedro María Alarcón-Elbal}

Tabla 2. Acciones físicas y biológicas que las familias pueden aplicar en cada tipo de depósito para evitar la presencia de culícidos en Jarabacoa, República Dominicana

\begin{tabular}{|c|c|c|c|c|c|c|c|c|c|c|}
\hline \multirow[b]{3}{*}{ Depósitos } & \multicolumn{10}{|c|}{ Acciones a ejecutar por parte de las familias } \\
\hline & \multicolumn{8}{|c|}{ Físicas } & \multirow{2}{*}{\begin{tabular}{|c|} 
Biológicas \\
$\begin{array}{c}\text { Aplicar } \\
\text { peces }\end{array}$
\end{tabular}} & \multirow[b]{2}{*}{$\begin{array}{c}\text { Total } \\
\text { acciones }\end{array}$} \\
\hline & Cepillar & Hermetizar & Flamear & $\begin{array}{l}\text { Piquetear } \\
\text { Aplastar }\end{array}$ & Virar & Quemar & $\begin{array}{c}\text { Colocar } \\
\text { bajo techo }\end{array}$ & Reutilizar & & \\
\hline \multicolumn{10}{|c|}{ Depósitos PU } & \\
\hline Tanque plástico & $\mathrm{X}$ & $\mathrm{X}$ & $\mathrm{X}$ & & $\mathrm{X}$ & & $\mathrm{X}$ & & $\mathrm{X}$ & 6 \\
\hline Galón plástico & & $\mathrm{X}$ & & & & & $\mathrm{X}$ & & & 2 \\
\hline Bebedero plástico & $\mathrm{X}$ & & $\mathrm{X}$ & & & & $\mathrm{X}$ & & & 3 \\
\hline Palangana plástica & $\mathrm{X}$ & & $\mathrm{X}$ & & $\mathrm{X}$ & & $\mathrm{X}$ & & & 4 \\
\hline Cubo plástico & $\mathrm{X}$ & & $\mathrm{X}$ & & $\mathrm{X}$ & & $\mathrm{X}$ & & & 4 \\
\hline Botellón plástico & & $\mathrm{X}$ & & & $\mathrm{X}$ & & $\mathrm{X}$ & & & 3 \\
\hline Ponchera plástica & $\mathrm{X}$ & & $\mathrm{X}$ & & $\mathrm{X}$ & & $\mathrm{X}$ & & & 4 \\
\hline $\begin{array}{l}\text { Escurridera } \\
\text { plástica }\end{array}$ & $\mathrm{X}$ & & & & & & & & & 1 \\
\hline Tragante cemento & \multicolumn{10}{|c|}{ Aplicarle agua y barrerlo cada siete días mínimo para evitar que se acumule el agua } \\
\hline Total & 6 & 3 & 5 & & 5 & & 7 & & 1 & 27 \\
\hline \multicolumn{10}{|c|}{ Depósitos NP } & \\
\hline $\begin{array}{l}\text { Neumáticos } \\
\text { usados }\end{array}$ & & & $\mathrm{X}$ & $\mathrm{X}$ & & & $\mathrm{X}$ & $\mathrm{X}$ & & 3 \\
\hline Bote plástico & & & & $\mathrm{X}$ & & & & $\mathrm{X}$ & & 2 \\
\hline Lata & & & & $\mathrm{X}$ & & & & $\mathrm{X}$ & & 2 \\
\hline Olla metal & $\mathrm{X}$ & & $\mathrm{X}$ & & $\mathrm{X}$ & & $\mathrm{X}$ & $\mathrm{X}$ & & 5 \\
\hline Bandeja metal & $\mathrm{X}$ & & & & & & & $\mathrm{X}$ & & 2 \\
\hline Bandeja plástica & $\mathrm{X}$ & & & & $\mathrm{X}$ & & $\mathrm{X}$ & $\mathrm{X}$ & & 4 \\
\hline Tapa plástica & $\mathrm{X}$ & & & & & & $\mathrm{X}$ & $\mathrm{X}$ & & 3 \\
\hline Botella plástica & & & & & $\mathrm{X}$ & & $\mathrm{X}$ & $\mathrm{X}$ & & 3 \\
\hline Lona plástica & $\mathrm{X}$ & & & & & & $\mathrm{X}$ & $\mathrm{X}$ & & 3 \\
\hline Cubo de metal & $\mathrm{X}$ & & $\mathrm{X}$ & & $\mathrm{X}$ & & $\mathrm{X}$ & $\mathrm{X}$ & & 5 \\
\hline Vaso cerámica & & & & & & & & $\mathrm{X}$ & & 1 \\
\hline Jarra metal & & & & & & & & $\mathrm{X}$ & & 1 \\
\hline Cilindro metal & & & & & & & & $\mathrm{X}$ & & 1 \\
\hline Tubería PVC & & & & & & & & $\mathrm{X}$ & & 1 \\
\hline Papelera plástica & & & & & & & & $\mathrm{X}$ & & 1 \\
\hline Aleta motor & & & & & & & & $\mathrm{X}$ & & 1 \\
\hline Maceta vidrio & \multirow{4}{*}{\multicolumn{10}{|c|}{$\begin{array}{l}\text { Revisión periódica que no exceda los siete días, } \\
\text { sobre todo en el período con incremento de lluvias }\end{array}$}} \\
\hline $\begin{array}{l}\text { Maceta de } \\
\text { cerámica }\end{array}$ & & & & & & & & & & \\
\hline Maceta plástica & & & & & & & & & & \\
\hline Planta & & & & & & & & & & \\
\hline Total & 6 & 0 & 3 & 2 & 4 & 0 & 7 & 16 & & 38 \\
\hline
\end{tabular}

Nota: en gris las acciones MoA en las que se crean condiciones permanentes desfavorables para la cría de vectores en sus hábitats.

Fuente: elaboración propia.

66 | Ciencia y Salud 2021; 5(3, septiembre-diciembre): 49-66 • Artículo original 